\title{
Circadian Regulation and Function of Voltage-Dependent Calcium Channels in the Suprachiasmatic Nucleus
}

\author{
Sang-Soep Nahm, ${ }^{1}$ Yuhua Z. Farnell, ${ }^{1}$ William Griffith, ${ }^{2}$ and David J. Earnest ${ }^{1,3}$ \\ Departments of ${ }^{1}$ Human Anatomy and Medical Neurobiology and ${ }^{2}$ Medical Pharmacology and Toxicology, College of Medicine, Texas A\&M University \\ Health Science Center, College Station, Texas 77843-1114, and ${ }^{3}$ Center for Biological Clocks Research, Department of Biology, Texas A\&M University, \\ College Station, Texas 77843-3258
}

Individual neurons within the suprachiasmatic nuclei (SCNs) are capable of functioning as autonomous clocks and generating circadian rhythms in the expression of genes that form the molecular clockworks. Limited information is available on how these molecular oscillations in individual clock cells are coordinated to provide for the ensemble rhythmicity that is normally observed from the entire SCN. Because calcium influx via voltage-dependent calcium channels (VDCCs) has been implicated in the regulation of gene expression and synchronization of rhythmicity across the population of SCN clock cells, we first examined the rat SCN and an immortalized line of SCN cells (SCN2.2) for expression and circadian regulation of different VDCC $\alpha 1$ subunits. The rat SCN and SCN2.2 cells exhibited mRNA expression for all major types of VDCC $\alpha 1$ subunits. Relative levels of VDCC expression in the rat SCN and SCN2.2 cells were greatest for L-type channels, moderate for P/Q- and T-type channels, and minimal for R-and N-type channels. Interestingly, both rat SCN and SCN2.2 cells showed rhythmic expression of P/Q- and T-type channels. VDCC involvement in the regulation of molecular rhythmicity in SCN2.2 cells was then examined using the nonselective antagonist, cadmium. The oscillatory patterns of $r$ Per 2 and $r B m a l 1$ expression were abolished in cadmium-treated SCN2.2 cells without affecting cellular morphology and viability. These findings raise the possibility that the circadian regulation of VDCC activity may play an important role in maintaining rhythmic clock gene expression across an ensemble of SCN oscillators.

Key words: clock; rhythm; SCN2.2; Per2; Bmal1; cadmium

\section{Introduction}

Circadian rhythms in mammals are controlled by the master pacemaker in the suprachiasmatic nucleus (SCN). In addition to orchestrating $24 \mathrm{~h}$ rhythmicity in other brain regions and peripheral systems, the SCN generates circadian oscillations in its biological activities independent of external input. SCN cells intrinsically produce circadian rhythms of neuropeptide secretion, cellular metabolism, and electrical activity in vivo and in vitro (Gillette, 1997). Oscillations in gene expression, especially those that serve as elements of the molecular clockworks, are another critical property of SCN cells (Panda et al., 2002). Specifically, Bmal1, Period1 (Per1), Per2, Cryptochrome1 (Cry1), Cry2, and $R e v$-erb $\alpha$ are rhythmically regulated through mutual interactions between their protein products, and this feedback loop forms the core of the circadian clock mechanism (Reppert and Weaver, 2002).

These oscillations in clock gene expression are not only an ensemble property of the entire nucleus but are also generated by individual SCN neurons. In brain slice preparations, the SCN

\footnotetext{
Received April 12, 2005; revised Aug. 22, 2005; accepted Aug. 31, 2005.

This work was supported by National Institutes of Health Program Project Grant P01 NS39546 (D.J.E.).

Correspondence should be addressed to Dr. David J. Earnest, Department of Human Anatomy and Medical Neurobiology, Texas A\&M University Health Science Center, 238 Reynolds Medical Building, College Station, TX 77843-1114. E-mail: dearnest@medicine.tamu.edu.

DOI:10.1523/JNEUROSCI.2733-05.2005

Copyright $\odot 2005$ Society for Neuroscience $\quad$ 0270-6474/05/259304-05\$15.00/0
}

exhibits ensemble rhythmicity in Per1-driven green fluorescent protein expression that is derived from the autonomous oscillations of individual neurons with multiphasic waveforms (Quintero et al., 2003). Identification of the processes responsible for the generation of molecular rhythmicity in individual cells and coordination of these oscillations across multiple cellular clocks is therefore of critical importance for understanding how SCN clock cells are coupled and function as a pacemaker that regulates circadian rhythms in other cells and tissues.

Recent findings support the involvement of ion channels that modulate membrane excitability/synaptic transmission in the regulation of molecular oscillations in clock cells. In Drosophila, abolishment of electrical activity in pacemaker neurons via targeted expression of specific ion channels disrupts circadian oscillations in PERIOD and TIMELESS levels (Nitabach et al., 2002). In mammals, there is increasing evidence that voltage-dependent calcium channels (VDCCs) may contribute to the clock function of SCN cells. SCN neurons exhibit the following: circadian rhythms of intracellular calcium concentration (Colwell, 2000; Ikeda et al., 2003), all major subtypes of VDCC currents (Cloues and Sather, 2003), and oscillations in the calcium currents generated by some VDCC subtypes (Pennartz et al., 2002; Kim et al., 2005). VDCCs have also been implicated in SCN intercellular communication, because treatment with cadmium, a nonselective calcium channel antagonist (Cloues and Sather, 2003), disrupts the synchronization of circadian rhythms in firing rate be- 
tween synaptically paired SCN neurons (Shirakawa et al., 2000). Consequently, the present study was conducted to further examine VDCC expression and function in the rat SCN. Immortalized rat SCN cells (SCN2.2) were analyzed in parallel, because these cells retain the endogenous rhythm-generating and pacemaker properties of the SCN in situ (Allen et al., 2004). The abundance of different VDCC subunits in the rat SCN and SCN2.2 cells was first determined and then their temporal patterns of expression were assessed for evidence of circadian regulation. To determine whether calcium channels are involved in regulating SCN circadian properties, we examined the effects of cadmium on molecular rhythmicity in SCN2.2 cells.

\section{Materials and Methods}

Animals and housing conditions. Adult male Sprague Dawley rats (150$200 \mathrm{~g}$; Harlan Laboratories, Indianapolis, IN) were housed two to three per cage. Access to food and water was provided ad libitum. All experimental procedures involving animals were approved by the University Laboratory Animal Care Committee at Texas A\&M University and performed in accordance with the National Institutes of Health Guide for the Care and Use of Laboratory Animals.

Cell culture conditions. SCN2.2 cells were cultured and propagated as described previously (Allen et al., 2004). In experiment 1, control cultures consisted of rat pituitary tumor cells (GH3) (CCL-82.1; American Type Culture Collection, Manassas, VA) that were maintained as described previously (Yonehara et al., 2001). During propagation of SCN2.2 and GH3 cells, the medium was changed at $48 \mathrm{~h}$ intervals, and cultures were split every 2-3 d at confluence.

Experiment 1. For in vivo experiments, animals were maintained for 3 weeks under a standard $12 \mathrm{~h}$ light/dark cycle (lights on at 6:00 A.M.). Beginning at the offset of this photoperiod [6:00 P.M. or circadian time (CT) 12], animals $(n=35)$ were exposed to constant darkness. Twelve hours later [6:00 A.M. (CT 0$)$ ], animals $(n=5)$ were killed under isoflurane anesthesia by decapitation at $6 \mathrm{~h}$ intervals for $36 \mathrm{~h}$ using an infrared viewer. After the eyes were removed in the dark, SCN tissue was immediately dissected as described previously (Earnest and Sladek, 1987). The entire cerebellum was also harvested. Tissue samples were frozen in liquid nitrogen and stored at $-80^{\circ} \mathrm{C}$. Individual samples were separately processed for extraction of total cellular RNA using the RNeasy Lipid Tissue RNA Extraction kit (Qiagen, Valencia, CA).

In vitro experiments were conducted using cultures of SCN2.2 and $\mathrm{GH} 3$ cells derived from a single passage. Beginning $36 \mathrm{~h}$ after plating on six-well plates (BD Biosciences, San Diego, CA), SCN2.2 cultures and control cultures containing GH3 cells $(n=3)$ were collected at $4 \mathrm{~h}$ intervals for $48 \mathrm{~h}$. Total RNA was extracted using the RNeasy Mini RNA extraction kit (Qiagen). For these in vitro experiments and in vivo analysis of the rat SCN and cerebellum, relative mRNA abundance of five representative VDCC $\alpha 1$ subunits in individual samples at each time point was determined by real-time PCR.

Experiment 2. To investigate the effects of general VDCC inhibition on clock gene expression, SCN2.2 cells were treated with the nonselective calcium channel antagonist, cadmium. Because exposure to cadmium at high concentrations $(0.1-1.0 \mathrm{~mm})$ is cytotoxic to rat neuronal cultures (Yoshida, 2001), $15 \mu \mathrm{M}$ cadmium was used in this experiment to avoid any long-term cytotoxicity. Although this concentration of cadmium has been shown to block VDCC currents in rat SCN neurons (Huang, 1993), we tested the effects of $15 \mu \mathrm{M}$ cadmium on VDCC currents in SCN2.2 cells to determine the degree of inhibition of whole-cell $\mathrm{Ba}^{2+}$ currents. Briefly, patch-clamp recordings were made as described previously (Griffith et al., 1994) with the exception that $5 \mathrm{~mm} \mathrm{Ba}^{2+}$ was used as the charge carrier. High voltage-activated (HVA) currents were generated using a voltage ramp protocol from -80 to $+50 \mathrm{mV}\left(V_{\mathrm{h}}=-60 \mathrm{mV}\right)$, and peak inward currents were measured.

SCN2.2 cells derived from a single passage were cultured on six-well plates and at confluence were either maintained in normal medium or treated with medium containing cadmium chloride (Sigma, St. Louis, $\mathrm{MO})$. Control and cadmium-treated cultures $(n=3)$ were harvested at $4 \mathrm{~h}$ intervals for $48 \mathrm{~h}$ to extract total RNA as described in experiment 1 .
The relative mRNA abundance of the clock genes, $r P e r 2$ and $r B m a l 1$, in individual samples at each time point was determined by real-time PCR. To assess the possibility that experimental observations may reflect the toxic effects of cadmium on SCN2.2 cells, the total cell number and viability were determined after 24 and $48 \mathrm{~h}$ of treatment using the trypan blue method.

Quantification of relative mRNA abundance was performed using TaqMan or SYBR-Green real-time PCR technology (Applied Biosystems, Foster City, CA) as described previously (Allen et al., 2004). To control for differences in sample RNA content, either cyclophilin A $(C y p A)$ mRNA or ribosomal RNA (rRNA) was amplified with the cDNA equivalent of $1 \mathrm{ng}$ of total RNA from the same samples. Each set of primers was evaluated to ensure equal amplification efficiencies and thus allow comparison of the relative mRNA levels among $\alpha 1$ subunits. The following probes and primers were designed with ABI PrimerExpress software: L-type (M67516), forward 5'-CCGGAAGCCAGTGCATTTT3', reverse 5'-TGGTGAAGATCGTGTCATTGACA-3', probe 5'-FAM-CCAAACAACAGGTTCCGCCTGCAGT-TAMRA-3'; P/Q-type (NM_012918), forward 5'-GGATGACAACACCGTTCACTTC-3', reverse 5'-CCACCCTTTGCGATTTTGAT-3', probe 5'-FAM-TGGCTCTGATCCGAACCGCCCTAMRA-3'; T-type (NM_031601.2), forward 5'-CCTGCCTGTTGCCGAGAG-3', reverse 5'-CAGGAGACGAAACCTTGACTGA-3', probe 5'-FAM-CGGCCTATATCTTTCCTC-TAMRA-3'; R-type (L15453), forward 5'-CGGGAGTGGAAGCGTCAT-3', reverse 5'-CCACCCTTTGCGATTTTGAT-3', probe 5'-FAM-TGGCTCTGATCCGAACCGCCC-TAMARA-3'; N-type (NM_147141), forward 5' -CAATTTCGAATACCTCACGCG-3', reverse 5' ${ }^{\prime}$-TGTAACTGATGCGCCCACAC-3', probe 5'-FAM- CTAGGGCCGCACCACCTCGATGA-TAMRA-3'; rPer2(AB016532), forward 5'-CCCATCCCACACTTGCCTC-3', reverse 5' -CACTGTGCCAGCCGGG-3'; rBmal1(AB012600), forward 5' GCAATCTGAGCTGCCTCGTT-3', reverse 5' -CCCGTATTTCCCCGTTCACT-3'; and rCyclophilin (M19533), forward 5'-TGTGCCAGGGTGGTGACTT-3', reverse 5' -TCAAATTTCTCTCCGTAGATGGACTT-3'.

The comparative $C_{\mathrm{T}}$ method described in the ABI Prism 7700 Sequence Detection System User Bulletin 2 was used to calculate the relative mRNA abundance for a given target gene. Using this method, the amount of target gene mRNA in each sample was normalized first to corresponding CypA mRNA or rRNA levels and then relative to a calibrator consisting of pooled cDNA from multiple samples that was analyzed on each reaction plate. For comparison of differences in gene expression over time, all values were adjusted in relation to time point 0 , which was set at an arbitrary unit of 1 .

Time-dependent fluctuations in VDCC and clock mRNA expression were identified by one-way ANOVA. Paired comparisons between peak values and those observed during the preceding or succeeding minimum were analyzed post hoc for statistical differences using the NewmanKeuls sequential range test.

\section{Results}

\section{Experiment 1}

To investigate the relative abundance and circadian regulation of VDCC mRNAs in the rat SCN and SCN2.2 cells, we chose five representative $\alpha 1$ subunits that constitute the pore-forming subunits for L-type ( $\alpha 1 \mathrm{C})$, N-type ( $\alpha 1 \mathrm{~B})$, P/Q-type ( $\alpha 1 \mathrm{~A})$, R-type $(\alpha 1 \mathrm{E})$, and T-type $(\alpha 1 \mathrm{G})$ calcium channels. Based on the comparative analysis described in the methods, all primers for the VDCC subtypes tested in this study showed similar amplification efficiencies, thereby enabling comparison of mRNA expression levels among different $\alpha 1$ subunits. For all five types of VDCC $\alpha 1$ subunits, mRNA expression was detected in both the rat SCN and SCN2. 2 cells using real-time PCR methods. These VDCC $\alpha 1$ subunits were differentially expressed in the rat SCN and SCN2.2 cells such that mRNA levels for L-type channels were substantially higher than those observed for all other VDCC phenotypes. Smaller differences in expression were observed among the other VDCC $\alpha 1$ subunits; in the rat SCN and SCN2.2 cells, levels of 

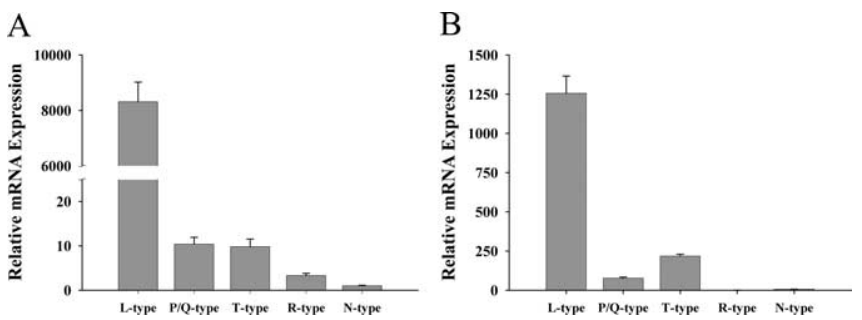

Figure 1. Differential expression of VDCC $\alpha 1$ subunits in the rat SCN $(\boldsymbol{A} ; n=5)$ and $\operatorname{SCN} 2.2$ $(\boldsymbol{B} ; n=3)$ cells. Bars denote determinations of mRNA abundance for L-type $(\alpha 1 \mathrm{C}), \mathrm{P} / \mathrm{Q}$-type $(\alpha 1 \mathrm{~A})$, T-type $(\alpha 1 \mathrm{G})$, R-type ( $\alpha 1 \mathrm{E})$, and $\mathrm{N}$-type ( $\alpha 1 \mathrm{~B})$ calcium channels by triplicate real-time $P C R$ analyses of RNA samples.

P/Q- and T-type calcium channels were higher relative to the expression of R- and N-type channels (Fig. 1A,B).

Analysis of the temporal patterns of VDCC mRNA levels revealed that expression of $\mathrm{P} / \mathrm{Q}$ - and T-type calcium channels varied rhythmically over the course of the circadian cycle in rat SCN and SCN2.2 cells (Fig. 2). Separate one-way ANOVAs indicated that determinations of P/Q- and T-type mRNA levels fluctuated significantly over time in both the rat SCN and SCN2.2 cells $(p<$ 0.001). In the rat SCN, expression of $\mathrm{P} / \mathrm{Q}$ - and T-type channels was low during the early subjective day, reached peak levels at CT 12 , and then declined during the late subjective night (Fig. 2A). For P/Q-type channels, expression in the rat SCN followed a similar pattern during the second cycle, but no significant differences were observed between peak and trough values.

SCN2.2 rhythms of VDCC expression in vitro were characterized by peak mRNA levels at 12 and $36 \mathrm{~h}$ for P/Q-type channels and at 8 and $32 \mathrm{~h}$ for T-type channels (Fig. 2 B). Peak expression of P/Q- and T-type calcium channels at these times in SCN2.2 cells was significantly greater $(p<0.05)$ than those observed during preceding or succeeding minima. For both rat SCN and SCN2.2 rhythms in the expression of P/Q- and T-type calcium channels, the difference between peak and minimum values ranged from 1.7-fold to 3.5-fold. In contrast, L-type channels, which represent the most abundant VDCC $\alpha 1$ subunit, as well as R-type subunits exhibited no evidence of circadian or even regular rhythmic fluctuations in the rat SCN and SCN2.2 cells (data not shown). The temporal pattern of $\mathrm{N}$-type calcium channel expression in the rat SCN and SCN2.2 cells was not determined because levels were consistently at or near limits of detection.

Similar to the SCN in vivo, the cerebellum exhibited circadian rhythms in the expression of P/Q- and T-type calcium channels. Separate one-way ANOVAs indicated that mRNA levels for P/Qand $\mathrm{T}$-type calcium channels varied significantly over time in the cerebellum $(p<0.001)$. For P/Q-type channels, cerebellar expression paralleled the temporal pattern observed in the SCN with peak levels occurring at CT 12 during the first cycle (Fig. $2 A)$. However, the cerebellar oscillation in the expression of T-type channels was phase delayed by $6 \mathrm{~h}$ relative to the SCN rhythm such that mRNA levels peaked during the subjective night at CT 18. Peak expression of P/Q- and T-type calcium channels at these times in the rat cerebellum was significantly greater $(p<0.05)$ than those observed during the preceding or succeeding minima. The rhythms in cerebellar expression of $\mathrm{P} / \mathrm{Q}$ - and T-type calcium channels were marked by $\sim 1.5$-fold differences between peak and minimum values. In contrast to SCN2.2 cells, GH3 cells showed increasing VDCC expression over the time in vitro but no sign of circadian or regular rhythmicity in VDCC mRNA levels (Fig. $2 B$ ).

\section{Experiment 2}

Because the roles of different VDCC subtypes in the regulation of SCN molecular rhythmicity are unknown, the nonselective calcium channel antagonist, cadmium, was used instead of specific antagonists to investigate the general effects of blocking a broad range of VDCC subtypes on clock gene expression. Whole-cell recordings from cultured SCN2.2 cells revealed that VDCC currents were almost completely blocked in all cells treated with 15 $\mu \mathrm{M}$ cadmium $(n=5)$ (Fig. $3 A)$. The amplitude of VDCC currents in SCN2.2 cells ranged from 40 to $120 \mathrm{pA}$, which is within the range of that reported for rat SCN neurons (Cloues and Sather, 2003). Similar to previous findings on calcium currents in rat SCN neurons (Pennartz et al., 2002), SCN2.2 cells exhibited HVA currents but not low-voltage activated (T-type) calcium currents perhaps because of the absence of T-type channels on the cell body.

Control cultures of SCN2.2 cells exhibited rhythmic patterns of $r$ Per 2 and rBmall mRNA abundance with twofold to threefold differences between peak and minimum levels and a peak-topeak interval of 20-24 h. For $r$ Per2, peak mRNA levels in control SCN2.2 cells were observed at 24 and $44 \mathrm{~h}$ (Fig. 3B). The rhythm in rBmall expression in control cells showed an anti-phase relationship to the rPer2 oscillation with peak mRNA abundance at 12 and $36 \mathrm{~h}$ (Fig. 3C). In cadmium-treated SCN2.2 cells, the relative levels of rPer 2 and rBmall mRNA were similar to those observed in control cultures. Statistical analyses revealed that cadmium-treated SCN2.2 cells exhibited no sign of circadian or even regular rhythmic fluctuations in rPer2 and rBmal1 expression. To assess the general effects of cadmium treatment on gene expression and to control for differences in RNA content between samples, rRNA expression was analyzed separately in control and cadmium-treated SCN2.2 cells. Throughout the course of the experiment, the relative levels of rRNA in cadmium-treated SCN2.2 cells at each time point were comparable with those observed in control cultures (data not shown), indicating that the reduced rPer2 and rBmal1 expression in SCN2.2 cells does not reflect a global decrease in gene expression but instead is a specific effect of cadmium treatment. SCN2.2 cells treated with cadmium for $48 \mathrm{~h}$ exhibited no palpable changes in cellular morphology, cell density, or cell viability. At the end of the $48 \mathrm{~h}$ treatment interval, the average cell density was 17,417 \pm 1412 cells $/ \mathrm{cm}^{2}$ with a viability of $94.4 \%$ in control cultures and $18,200 \pm 700$ cells $/ \mathrm{cm}^{2}$ with a viability of $94.6 \%$ in cadmiumtreated cells $(p=0.74)$.

\section{Discussion}

The present results demonstrate that all types of $\alpha 1$ subunits, which form the major calcium-conducting pore of VDCC (Catterall, 2000), are expressed in both the rat SCN and SCN2.2 cells. This observation is consistent with pharmacological evidence for functionality of L-, N-, P/Q-, R-, and T-type calcium currents in rat SCN neurons (Pennartz et al., 2002; Cloues and Sather, 2003; Kim et al., 2005). The relative expression of these different VDCC subtypes was similar in the rat SCN and SCN2.2 cells with a general pattern in which L-type channels were the most abundant, T- and P/Q-type levels were intermediate, and R- and $\mathrm{N}$-type channels were minimal. The relative proportions of $\mathrm{T}$ and $\mathrm{P} / \mathrm{Q}$-type channels were not fully congruent in the rat SCN and SCN2.2 cells, but this disparity may reflect differences in the degree of cellular heterogeneity between these experimental models. Although SCN2.2 cells were developed as a heterogeneous, rather than clonal, cell line to provide broad representation of SCN phenotypes, the immortalization strategy used to 
A
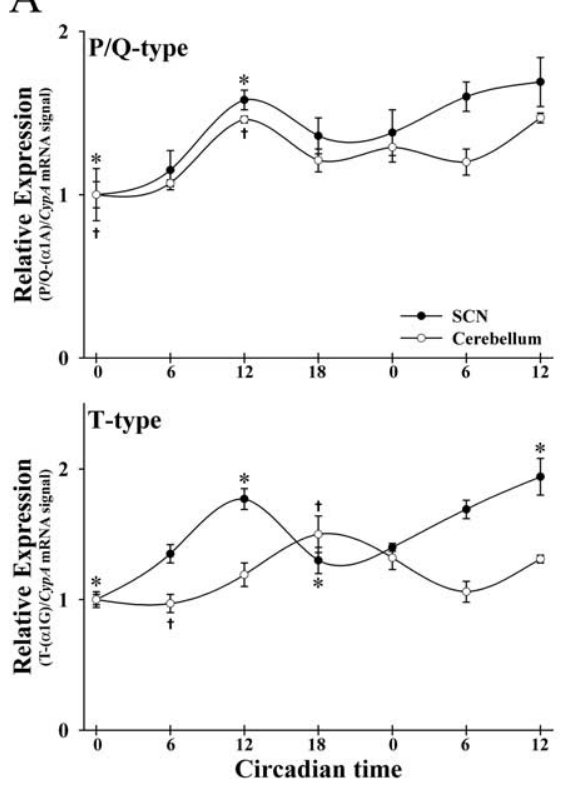

B
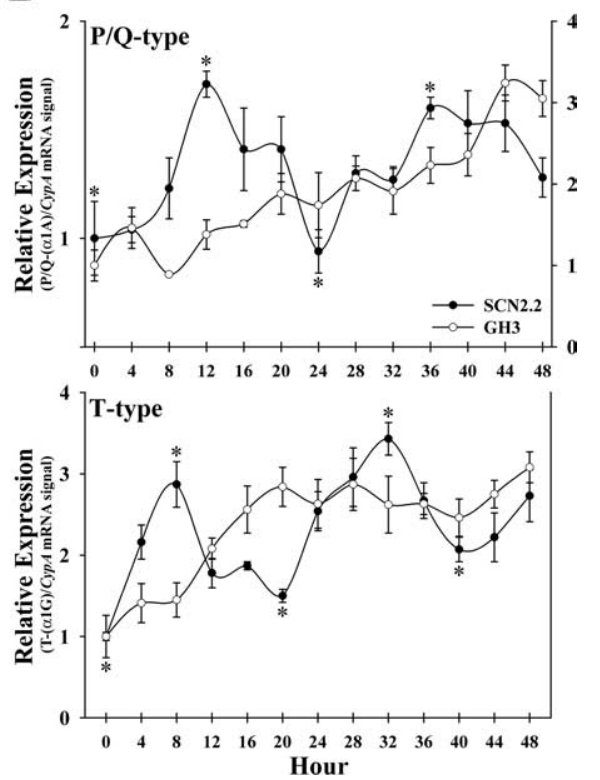

Figure 2. Circadian regulation of VDCC expression in vivo and in vitro. Temporal patterns of P/Q- and T-type calcium channel expression in the rat SCN and cerebellum $(\boldsymbol{A} ; n=5)$ and in SCN2.2 and GH3 cells $(\boldsymbol{B} ; n=3)$. Symbols denote the ratios of P/Q-type $(\alpha 1 \mathrm{~A})$ or T-type $(\alpha 1 \mathrm{G})$ subunit/CypA mRNA signal. For GH3 cells, P/Q-type calcium channel expression was plotted relative to the secondary ordinant $(\boldsymbol{B})$. Significant differences between peak mRNA levels for $\mathrm{P} / \mathrm{Q}$ - and T-type calcium channels and those observed during preceding or succeeding minima $(p<0.05)$ are denoted separately for the SCN $\left(^{*}\right)$ and cerebellum $(\dagger)$.

establish this line may have nonspecifically selected for or against certain cell types and thereby narrowed the breadth of SCN2.2 gene expression.

Transcriptional regulation of VDCC is primarily unknown, especially with regard to whether these channels are rhythmically expressed in the SCN and in other brain regions. In the context of the present study, it is interesting that neurotrophic factors have been shown to regulate neuronal VDCC expression (Baldelli et al., 1999). In cultured motor neurons, brain-derived neurotrophic factor (BDNF) selectively induces an upregulation of P- and R-type but not L- or N-type calcium channels. Because the increase in P-and R-type currents was maximal after $12 \mathrm{~h}$ of BDNF treatment, these findings raise the possibility that rhythmic BDNF expression within SCN and SCN 2.2 cells (Liang et al., 1998; Earnest et al., 1999) may play a role in the regulation of $\mathrm{P} / \mathrm{Q}$-type calcium channel expression and perhaps calcium currents in the SCN.
Although their specific roles have not been fully defined, previous studies suggest that some VDCC subtypes may be involved in regulating SCN neural activity, circadian behavior, and sleep. The observed rhythm in SCN expression of T-type channels is interesting, because these calcium channels appear to be involved in the mechanism for the spontaneous firing activity of SCN neurons (Akasu et al., 1993), and T-type calcium currents show circadian variation in retinorecipient neurons within the SCN (Kim et al., 2005). Recent studies indicate that T-type calcium channels also mediate glutamate-induced phase shifts of the clock mechanism (Kim et al., 2005). T-type channels have also been implicated in sleep regulation based on the observation that mice lacking the $\alpha 1 \mathrm{G}$ subunit of this calcium channel subtype exhibit altered sleep oscillations with increased frequency of brief awakening during nonrapid eye movement episodes (Lee et al., 2004). Despite their basal levels and arrhythmic pattern of expression, R- and $\mathrm{N}$-type calcium channels may have some functional importance in the SCN. R-type calcium currents regulate afterhyperpolarization, a process that modulates spontaneous firing rate in SCN neurons (Cloues and Sather, 2003), and intraventricular infusion of a N-type channel antagonist abolishes the circadian rhythm of drinking behavior in rats (Masutani et al., 1995).

The present finding that cadmium treatment disrupts rhythmic rPer2 and rBmal1 expression in SCN2.2 cells suggests that calcium channels may have an important function in coordinating rhythmic clock gene expression across an ensemble of cellautonomous oscillators. Because $15 \mu \mathrm{M}$ cadmium treatment blocked most VDCC currents in SCN 2.2 cells, these cadmiuminduced disturbances in clock gene rhythms are most likely attributable to blocking high-voltage-dependent calcium channels, including L-type or P/Q-type calcium channels. One possible explanation for the disruption of clock gene oscillations in cadmium-treated SCN2.2 cells is that these calcium channels may play key roles in the synchronization or intercellular coupling of
A

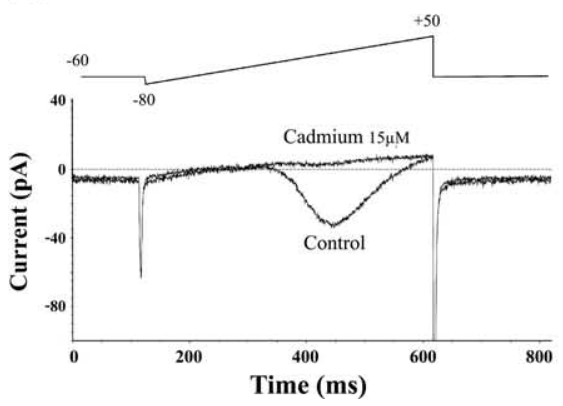

B

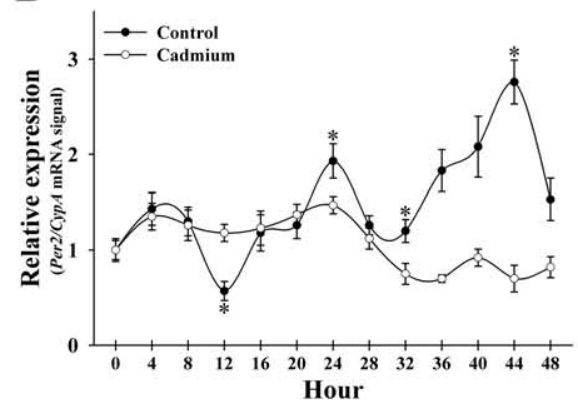

C

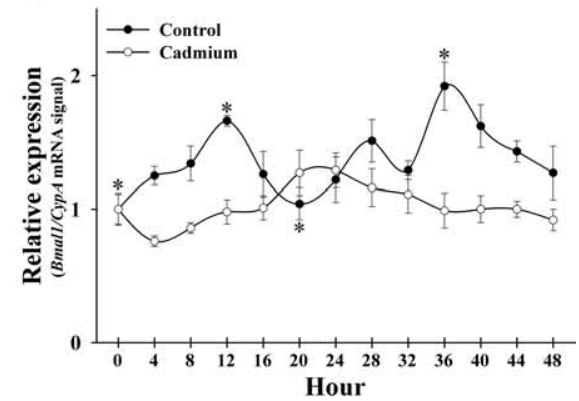

Figure 3. A, Cadmium blocks voltage-dependent calcium currents in SCN 2.2 cells. Whole-cell patch-clamp recordings of a ramp current in a SCN2.2 cell before and after bath application of $15 \mu \mathrm{M}$ cadmium. Cadmium disrupts the rhythms of $r$ Per 2 ( $\boldsymbol{B}$ ) and $r$ Bmal 1 ( $($ ) expression in SCN2.2 cells. Temporal profiles of $r$ Per 2 and $r$ Bmal $1 \mathrm{mRNA}$ expression in control and cadmium-treated SCN2.2 cells $(n=3)$. Symbols denote the ratios of $r P e r 2$ and $r B m a l 1 / C y p A$ mRNA signal. Asterisks indicate significant differences between peak $r$ Per 2 or $r B m a l 1$ expression and that observed during preceding or succeeding minima $(p<0.05)$. 
clock cell populations. Decreased coupling strength between multiple oscillators is thought to produce deviations in rhythm amplitude or period length from the population mean and thereby yield irregular ensemble rhythmicity (Enright, 1980). Thus, the observed loss of rhythmicity in rPer2 and rBmall expression may be a result of the effects of cadmium on VDCC function in the coordination of molecular oscillations among populations of SCN2.2 clock cells. This possibility is supported by evidence indicating that VDCCs are involved in the electrical coupling between SCN neurons. In dissociated SCN cultures, intercellular spike interval between synaptically paired neurons was significantly increased during treatment with cadmium (Shirakawa et al., 2000). As determined in the present study, circadian regulation of P/Q-type calcium channels in SCN cells is compatible with their potential involvement in intercellular coupling and coordination of molecular oscillations between SCN clock cells.

An alternative explanation for the observed effects of cadmium on molecular oscillations in SCN2.2 cells is that VDCCs may be involved in regulating clock gene transcription. Previous studies indicate that L-type calcium currents induce transcription of immediate early genes, cAMP responsive elementbinding protein (CREB), and other kinases through their regulation of synaptic transmission and calcium influx (Murphy et al., 1991; Dolmetsch et al., 2001). In conjunction with evidence for the independent activation of the Per genes by CREB-dependent signaling (Travnickova-Bendova et al., 2002), these findings raise the possibility that cadmium inhibition of L-type channels and other VDCCs may affect calcium signaling-dependent activation of gene transcription, thereby altering clock gene oscillations in SCN2.2 cells. Although it will be difficult to discriminate between VDCC involvement in the synchronization of clock cell populations and the regulation of clock gene expression, additional analysis using specific antagonists may yield important insight into the importance of different calcium channel subtypes in the regulation of SCN molecular oscillations.

\section{References}

Akasu T, Shoji S, Hasuo H (1993) Inward rectifier and low-threshold calcium currents contribute to the spontaneous firing mechanism in neurons of the rat suprachiasmatic nucleus. Pflügers Arch 425:109-116.

Allen GC, Farnell Y, Bell-Pedersen D, Cassone VM, Earnest DJ (2004) Effects of altered Clock gene expression on the pacemaker properties of SCN2.2 cells and oscillatory properties of NIH/3T3 cells. Neuroscience 127:989-999.

Baldelli P, Magnelli V, Carbone E (1999) Selective up-regulation of P- and R-type $\mathrm{Ca}^{2+}$ channels in rat embryonic motoneurons by BDNF. Eur J Neurosci 11:1127-1133.

Catterall WA (2000) Structure and regulation of voltage-gated $\mathrm{Ca}^{2+}$ channels. Annu Rev Cell Dev Biol 16:521-555.

Cloues RK, Sather WA (2003) Afterhyperpolarization regulates firing rate in neurons of the suprachiasmatic nucleus. J Neurosci 23:1593-1604.

Colwell CS (2000) Circadian modulation of calcium levels in cells in the suprachiasmatic nucleus. Eur J Neurosci 12:571-576.

Dolmetsch RE, Pajvani U, Fife K, Spotts JM, Greenberg ME (2001) Signal- ing to the nucleus by an L-type calcium channel-calmodulin complex through the MAP kinase pathway. Science 294:333-339.

Earnest DJ, Sladek CD (1987) Circadian vasopressin release from perfused rat suprachiasmatic explants in vitro: effects of acute stimulation. Brain Res 422:398-402.

Earnest DJ, Liang F-Q, Ratcliff M, Cassone VM (1999) Immortal time: circadian clock properties of cell lines derived from the rat suprachiasmatic nucleus. Science 283:693-695.

Enright JT (1980) Temporal precision in circadian systems: a reliable neuronal clock from unreliable components? Science 209:1542-1545.

Gillette MU (1997) Cellular and biochemical mechanisms underlying circadian rhythms in vertebrates. Curr Opin Neurobiol 7:797-804.

Griffith WH, Taylor L, Davis MJ (1994) Whole-cell and single-channel calcium currents in guinea pig basal forebrain neurons. J Neurophysiol 71:2359-2376.

Huang RC (1993) Sodium and calcium currents in acutely dissociated neurons from rat suprachiasmatic nucleus. J Neurophysiol 70:1692-1703.

Ikeda M, Sugiyama T, Wallace CS, Gompf HS, Yoshioka T, Miyawaki A, Allen CN (2003) Circadian dynamics of cytosolic and nuclear $\mathrm{Ca}^{2+}$ in single suprachiasmatic nucleus neurons. Neuron 38:253-263.

Kim DY, Choi HJ, Kim JS, Kim SK, Jeong DU, Shin HC, Kim MJ, Han H-C, Hong SK, Kim YI (2005) Voltage-gated calcium channels play crucial roles in the glutamate-induced phase shifts of the rat suprachiasmatic circadian clock. Eur J Neurosci 21:1215-1222.

Lee J, Kim D, Shin HS (2004) Lack of delta waves and sleep disturbances during non-rapid eye movement sleep in mice lacking alpha1G-subunit of T-type calcium channels. Proc Natl Acad Sci USA 101:18195-18199.

Liang F-Q, Walline R, Earnest DJ (1998) Circadian rhythm of brain-derived neurotrophic factor in the rat suprachiasmatic nucleus. Neurosci Lett 242:89-92.

Masutani H, Matsuda Y, Nagai K, Nakagawa H (1995) Effect of $\Omega$-conotoxin, a calcium channel blocker, on the circadian rhythm in rats. Biol Rhythm Res 26:573-581.

Murphy TH, Worley PF, Baraban JM (1991) L-type voltage-sensitive calcium channels mediate synaptic activation of immediate early genes. Neuron 7:625-635.

Nitabach MN, Blau J, Holmes TC (2002) Electrical silencing of Drosophila pacemaker neurons stops the free-running circadian clock. Cell 109:485-495.

Panda S, Antoch MP, Miller BH, Su AI, Schook AB, Straume M, Schultz PG, Kay SA, Takahashi JS, Hogenesch JB (2002) Coordinated transcription of key pathways in the mouse by the circadian clock. Cell 109:307-320.

Pennartz CM, de Jeu MT, Bos NP, Schaap J, Geurtsen AM (2002) Diurnal modulation of pacemaker potentials and calcium current in the mammalian circadian clock. Nature 416:286-290.

Quintero JE, Kuhlman SJ, McMahon DG (2003) The biological clock nucleus: a multiphasic oscillator network regulated by light. J Neurosci 23:8070-8076.

Reppert SM, Weaver DR (2002) Coordination of circadian timing in mammals. Nature 418:935-941.

Shirakawa T, Honma S, Katsuno Y, Oguchi H, Honma KI (2000) Synchronization of circadian firing rhythms in cultured rat suprachiasmatic neurons. Eur J Neurosci 12:2833-2838.

Travnickova-Bendova Z, Cermakian N, Reppert SM, Sassone-Corsi P (2002) Bimodal regulation of mPeriod promoters by CREB-dependent signaling and CLOCK/BMAL1 activity. Proc Natl Acad Sci USA 99:7728-7733.

Yonehara T, Kanasaki H, Yamamoto H, Fukunaga K, Miyazaki K, Miyamoto E (2001) Involvement of mitogen-activated protein kinase in cyclic adenosine $3^{\prime}, 5^{\prime}$-monophosphate-induced hormone gene expression in rat pituitary GH(3) cells. Endocrinology 142:2811-2819.

Yoshida S (2001) Re-evaluation of acute neurotoxic effects of $\mathrm{Cd}^{2+}$ on mesencephalic trigeminal neurons of the adult rat. Brain Res 892:102-110. 